\title{
Experimental Evidence for a Large Collinear Electric Field Gradient of Ir in hcp-Co
}

\author{
G. Seewald, B. Hinfurtner, E. Hagn, and E. Zech \\ Physik-Department, Technische Universität München, D-85748 Garching, Germany \\ D. Forkel-Wirth and R. Eder \\ PPE Division, CERN, CH-1211 Geneva 23, Switzerland
}

ISOLDE Collaboration

CERN, CH-1211 Geneva 23, Switzerland

(Received 21 October 1997)

\begin{abstract}
Measurements of quadrupole-interaction-resolved NMR on oriented nuclei were performed on ${ }^{188} \mathrm{Ir}$ in a hcp-Co single crystal. The magnetic and electric hyperfine interaction frequencies were determined as function of the angle $\theta$ between the electronic magnetization $\vec{M}$ and the single crystal $c$ axis. A large collinear electric field gradient (CEFG) (principal axis parallel $\vec{M}$ ) was found, $e q^{(c)}=-0.378(7) \times 10^{17} \mathrm{~V} / \mathrm{cm}^{2}$. Within the series of the $5 d$ elements $\mathrm{Ir}, \mathrm{Pt}$, $\mathrm{Au}$, and $\mathrm{Hg}$ this is exceptional: The experimental CEFG's of $\mathrm{Pt}$, $\mathrm{Au}$, and $\mathrm{Hg}$ in hcp-Co are at least $\sim 2$ orders of magnitude smaller and consistent with $e q^{(c)}=0 . \quad$ [S0031-9007(98)05894-3]

PACS numbers: 76.60.Jx, 75.50.Bb, 76.60.Gv, 76.80.+y
\end{abstract}

It is well known that an electric field gradient (EFG) exists at the nuclear site of atoms dissolved as dilute impurities in the cubic ferromagnetic hosts $\mathrm{Fe}, \mathrm{Ni}$, and fcc-Co [1-10]. The existence of an EFG in a cubic ferromagnetic host lattice has been attributed to an unquenched orbital momentum caused by the spin-orbit interaction of the $n d$ electrons $[4,8,9]$. The principal axes system of this EFG is given by the direction of the magnetization; the electric and magnetic hyperfine interactions are thus collinear. Especially for $5 d$ elements the collinear EFG (CEFG) $e q^{(c)}$ is relatively large. Experimental data exist for $\operatorname{Ir} F e$ [1,4,10], $\mathrm{IrNi}$ [1,4], AuFe [5], AuNi [6], $\mathrm{AuCo}^{(\mathrm{fcc})}$ [7], $\mathrm{PtFe}$ [6], and $\mathrm{PtCo}{ }^{\left(\mathrm{fcc}^{2}\right)}[6]$. As the CEFG originates from the spin-orbit interaction of the $n d$ electrons at the impurity site, such a CEFG could exist also for $n d$ elements as dilute impurities in hcp-Co. In hcp-Co, however, a relatively large EFG exists because of the noncubic lattice structure, which we denote in the following as lattice EFG (LEFG) $e q^{(L)}$. Both contributions can be separated due to the different transformation properties against rotation of the magnetization $\vec{M}$ with respect to the crystal axis $c$. Whereas the CEFG is (to first approximation) invariant against rotation of the magnetization, the LEFG has second-order-tensor transformation properties. Actually, for ${ }^{59} \mathrm{CoCo} o^{(\mathrm{hcp})}$ the existence of a small rotation-invariant EFG has been reported in the literature [11,12]. Thus, taking into account that the CEFG's of $5 d$ elements in $\mathrm{Fe}$ and $\mathrm{Ni}$ are by $\sim 2$ orders of magnitude larger than those of the $3 d$ elements, a moderately large CEFG was to be expected for $5 d$ elements in hcp-Co. First experiments on ${ }^{198} \mathrm{AuCo} o^{(\mathrm{hcp})}$, however, gave evidence that the CEFG for $\mathrm{Au}$ in hcp-Co is extremely small and consistent with zero, $e q^{(c)}\left(\mathrm{AuCo} o^{(\mathrm{hcp})}\right)=-0.002(3) \times 10^{17} \mathrm{~V} / \mathrm{cm}^{2}$ [13]. Comparing this with the CEFG of $\mathrm{Au}$ in $\mathrm{Fe}$ and
fcc-Co, $e q^{(c)}(\mathrm{AuFe})=-0.113(5) \times 10^{17} \mathrm{~V} / \mathrm{cm}^{2} \quad$ [5], $e q^{(c)}\left(\mathrm{AuCo} o^{(\mathrm{fcc})}\right)=(-) 0.11(3) \times 10^{17} \mathrm{~V} / \mathrm{cm}^{2} \quad$ [7], one might conclude that the CEFG of Au in hcp-Co is zero because of a symmetry of the local electronic wave function. In order to clarify whether the CEFG's are comparably small for all $5 d$ elements, we performed measurements on $\mathrm{Pt}, \mathrm{Hg}$, and $\mathrm{Ir}$ in hcp-Co. Actually, for $\mathrm{Pt}$ and $\mathrm{Hg}$ in hcp$\mathrm{Co}$ a similar behavior was found as for $\mathrm{Au}$ : The CEFG is - if at all existent - extremely small and consistent with zero, too: $\left|e q^{(c)}\left(\mathrm{PtC} o^{(\mathrm{hcp})}\right)\right| \leq 0.008 \times 10^{17} \mathrm{~V} / \mathrm{cm}^{2}$, $\left|e q^{(c)}\left(\mathrm{HgCo} o^{(\mathrm{hcp})}\right)\right| \leq 0.013 \times 10^{17} \mathrm{~V} / \mathrm{cm}^{2}$ [6]. For Ir in hcp-Co, however, now completely unexpected, a very large CEFG was found. These experiments are presented here.

The hyperfine interaction of radioactive isotopes at substitutional lattice sites in ferromagnetic hcp-Co consists of a magnetic-dipole and an electric-quadrupole contribution:

(i) The magnetic hyperfine field $B_{\mathrm{HF}}$ is parallel (antiparallel) to the electronic magnetization $\vec{M}$, the absolute magnitude depending (slightly) on the angle between $\vec{M}$ and the single-crystal $c$ axis. The magnetic interaction frequency is given by

$$
\begin{gathered}
\nu_{\mathrm{mag}}=\left|g \mu_{N} / h\right|\left|\vec{B}_{\mathrm{HF}}+\vec{B}_{0}\right|, \\
\vec{B}_{0}=\vec{B}_{\mathrm{ext}}-\vec{B}_{\mathrm{dem}},
\end{gathered}
$$

where $B_{\text {ext }}$ is the applied external magnetic field, $B_{0}$ is the (effective) external magnetic field at the nuclear site, and $B_{\text {dem }}$ is a demagnetization field, which depends on the geometry of the sample.

(ii) Because of the hexagonal symmetry of hcp-Co, an axially symmetric lattice electric field gradient (LEFG) $e q^{(L)}$ exists, the principal axis being given by the singlecrystal $c$ axis. In addition, a CEFG $e q^{(c)}$ may exist, the 
principal axis being given by the direction of $\vec{B}_{\mathrm{HF}}$. The strength of the electric interaction is described by the quadrupole interaction frequencies $\nu_{Q}^{(c, L)}$

$$
\nu_{Q}^{(c, L)}=e^{2} q^{(c, L)} Q / h,
$$

where $e Q$ is the nuclear spectroscopic quadrupole moment. For $\operatorname{IrFe}$, it was found recently that the CEFG depends on the direction of magnetization with respect to the crystallographic axes [10]. Such a directional dependence of the CEFG might also occur for hcp-Co; it is - if existent-in first order described by

$$
\nu_{Q}^{(c)}=\nu_{Q}^{(c, \text { iso })}+\nu_{Q}^{(c, \text { anis })} P_{2}(\cos \theta),
$$

where $\theta$ is the angle between the electronic magnetization $\vec{M}$ and the single crystal $c$ axis.

In the absence of an external magnetic field $B_{\text {ext }}$, or if $B_{\text {ext }}$ is applied parallel $c, \vec{M}$ and $\vec{B}_{\mathrm{HF}}$ are parallel to the $c$ axis, which is the direction of spontaneous magnetization. We denote this as " 0 " geometry." By applying an external magnetic field $B_{\text {ext }}$ perpendicular to the $c$ axis (" $90^{\circ}$ geometry"), the direction of the magnetization can be turned away from the $c$ axis $\left(B_{\text {ext }}=0\right)$ towards the direction of the external magnetic field, which is reached for $B_{0} \geq B_{A}^{\perp}\left(B_{\text {ext }} \geq B_{A}^{\perp}+B_{\text {dem }}\right)$, where $B_{A}^{\perp}=$ $13.5 \mathrm{kG}$ is the perpendicular anisotropy field. The angle $\theta$ between $\vec{M}$ and $c$ can be adjusted between $0^{\circ}$ and $90^{\circ}$ via $B_{\text {ext }}$ : It is obtained from the minimum of the total energy

$$
E_{\mathrm{tot}}=K_{1} \sin ^{2} \theta+K_{2} \sin ^{4} \theta-\vec{M}(\theta) \vec{B}_{0},
$$

where $K_{1}$ and $K_{2}$ are the known anisotropy constants for Co.

The energy eigenvalues $E_{m}$ of the nuclear spin system are calculated by diagonalization of the Hamiltonian,

$$
\begin{aligned}
\mathcal{H} & =\mathcal{H}_{M}+\mathcal{H}_{Q}^{(c)}+\mathcal{D}(\theta) \mathcal{H}_{Q}^{(L)} \mathcal{D}^{-1}(\theta), \\
\mathcal{H}_{M} & =-h \nu_{\text {mag }} I_{z}, \\
\mathcal{H}_{Q}^{(c, L)} & =h \nu_{Q}^{(c, L)} \frac{3 I_{z}^{2}-I(I+1)}{4 I(2 I-1)},
\end{aligned}
$$

where $\mathcal{D}(\theta)$ is the normally used rotation matrix and $I$ is the nuclear spin. A set of $2 I$ subresonance frequencies $\nu_{m \rightarrow m+1}=\left|E_{m+1}-E_{m}\right| / h$ is obtained.

In the following we confine ourselves to $I=1\left({ }^{188} \mathrm{Ir}\right)$. Then two subresonances with frequencies $\nu_{1}$ and $\nu_{2}$ exist. Let us, for a simplified discussion, first assume that the lattice quadrupole interaction is small compared to the

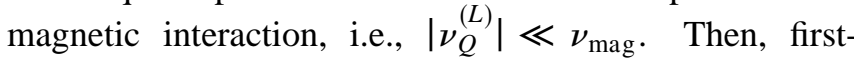
order perturbation theory yields that the effective lattice quadrupole interaction follows a $P_{2}(\cos \theta)$ dependence:

$$
\begin{gathered}
\nu_{1}=\nu_{\mathrm{mag}}(\theta)-\frac{1}{2} \Delta \nu_{Q}(\theta), \\
\nu_{2}-\nu_{1}=\Delta \nu_{Q}(\theta)=\frac{3}{2}\left[\nu_{Q}^{(\mathrm{iso})}+\nu_{Q}^{(\mathrm{anis})} P_{2}(\cos \theta)\right],
\end{gathered}
$$

$$
\begin{gathered}
\nu_{Q}^{(\text {iso })}=\nu_{Q}^{(c, \text { iso })}, \\
\nu_{Q}^{(\text {anis })}=\nu_{Q}^{(L)}+\nu_{Q}^{(c, \text { anis })} .
\end{gathered}
$$

Experimentally, $\nu_{Q}^{(\text {iso })}$ and $\nu_{Q}^{\text {(anis) }}$ are best obtained from measurements of $\Delta \nu_{Q}(\theta)$ for $\theta=0^{\circ}\left(\Delta \nu_{Q}^{\|}\right)$and $90^{\circ}$ $\left(\Delta \nu_{Q}^{\perp}\right)$. Actually, as $\left|\nu_{Q}^{(L)}\right| \ll \nu_{\text {mag }}$ is not fulfilled here, there is a small deviation from the $P_{2}(\cos \theta)$ dependence of the effective lattice quadrupole interaction. In the data analysis this has been taken into account properly. In the absence of a CEFG, $\Delta \nu_{Q}^{\|} / \Delta \nu_{Q}^{\perp}=-2$ is expected.

In the NMR-ON method the resonant absorption of an applied radio frequency (rf) field is detected via nuclear radiation [14]. The angular distribution $W(\vartheta)$ of $\gamma$ rays emitted in the decay of oriented nuclei at the temperature $T$ is given by

$$
W(\vartheta)=1+\sum_{k \text { even }} A_{k} B_{k}\left(a_{m}\right) P_{k}(\cos \vartheta) Q_{k},
$$

where all parameters have their conventional meaning [15]. In the present work the "anisotropy"

$$
\epsilon=W\left(0^{\circ}\right) / W\left(90^{\circ}\right)-1
$$

has been analyzed.

Samples of ${ }^{188} \mathrm{IrCo}{ }^{(\mathrm{hcp})}$ were prepared at the massseparator ISOLDE/CERN. ${ }^{188} \mathrm{Hg}$, obtained from the spallation reaction of $1 \mathrm{GeV}$ protons on a liquid $\mathrm{Pb}$ target, was implanted with $E=60 \mathrm{keV}$ into a hcp-Co single crystal disk $(\Phi=10 \mathrm{~mm}$; thickness $0.25 \mathrm{~mm}$; $c$ axis parallel to the surface). The following decay chain is relevant:

$$
\begin{aligned}
& { }^{188} \mathrm{Hg}\left(T_{1 / 2}=3 \mathrm{~min}\right) \rightarrow{ }^{188} \mathrm{Au}\left(T_{1 / 2}=9 \mathrm{~min}\right) \\
& \rightarrow{ }^{188} \mathrm{Pt}\left(T_{1 / 2}=10.2 d\right) \rightarrow{ }^{188} \mathrm{Ir}\left(T_{1 / 2}=41.5 h\right) \rightarrow{ }^{188} \mathrm{Os} .
\end{aligned}
$$

Using a ${ }^{3} \mathrm{He}-{ }^{4} \mathrm{He}$-dilution refrigerator with a top-loading facility, the samples were cooled to temperatures near $10 \mathrm{mK}$.

For $0^{\circ}$ geometry, measurements were performed for ten different values of $B_{\text {ext }}$ between 0 and $20 \mathrm{kG}$. Figure 1 shows both subresonances $\nu_{1}$ and $\nu_{2}$ as measured at $B_{\text {ext }}=10 \mathrm{kG}$. The resonance shift of both resonances was in perfect agreement with the expectation according to the known $g$ factor. The subresonance separation was-as expected-independent on $B_{\text {ext }}$; the final average value is

$$
\Delta \nu_{Q}^{\|}=-38.08(1) \mathrm{MHz} .
$$

The top part of Fig. 2 shows the result of a $90^{\circ}$ geometry measurement for $B_{\mathrm{ext}}=18 \mathrm{kG}$, from which $\Delta \nu_{Q}^{\perp}=+9.06(12) \mathrm{MHz}$ is obtained. Thus $\Delta \nu_{Q}^{\|} / \Delta \nu_{Q}^{\perp}=$ $-4.20(6)$, which is strongly different from -2 as expected for a pure lattice quadrupole interaction.

In principle, a deviation of $\Delta \nu_{Q}^{\|} / \Delta \nu_{Q}^{\perp}$ from -2 could be due to an angular dependence of the principal component of the LEFG. This could be due to an angular dependence of the Sternheimer effect. (The Sternheimer 


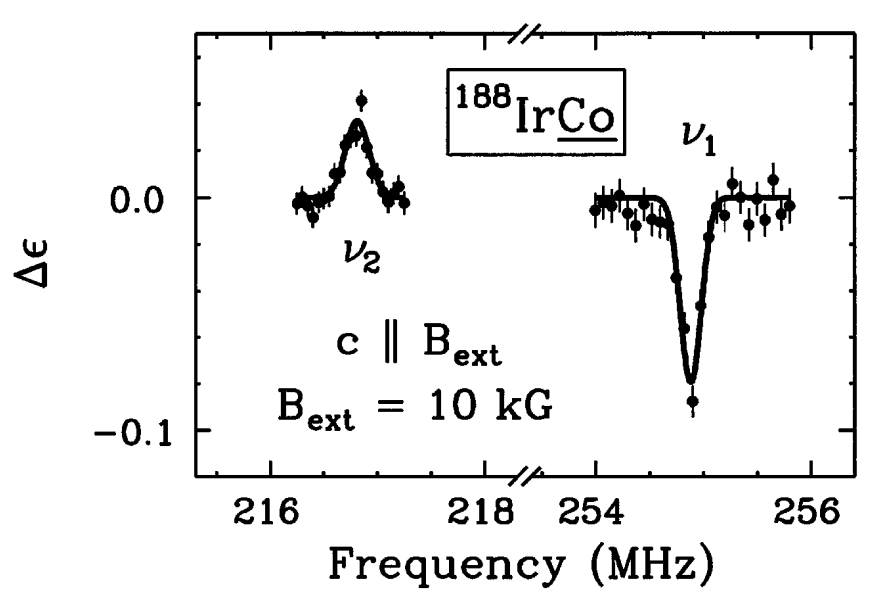

FIG. 1. NMR-ON spectra of ${ }^{188} \mathrm{IrC} o^{(\mathrm{hcp})}$ measured in $0^{\circ}$ geometry. For $\nu_{1}$ the $\gamma$ anisotropy is reduced, whereas it is enhanced for $\nu_{2}$. The latter is due to the population transfer from the $m=0$ sublevel to the energetically higher $m=1$ sublevel. Thus, with respect to thermal equilibrium, the sum of populations of the $m=+1$ and -1 states is enhanced by the resonance absorption at $\nu_{2}$, which is accompanied by an increase of the $\gamma$ anisotropy.

factor takes into account the (anti)shielding of an external EFG - here the LEFG - by the local electrons at the impurity site.) Then, $\nu_{Q}^{(L)}$ in Eq. (10) has to be replaced by

$$
\nu_{Q}^{(L)}(\theta)=\nu_{Q}^{(L)}\left[1+a_{2}^{(L)} P_{2}(\cos \theta)\right],
$$

where $a_{2}^{(L)}$ is an anisotropy constant for the LEFG.

Thus, there are two extreme scenarios within which a deviation of $\Delta \nu_{Q}^{\|} / \Delta \nu_{Q}^{\perp}$ from -2 can be explained, the existence of a large CEFG or an angular dependence of $\nu_{Q}^{(L)}$. These can be distinguished by a measurement of $\Delta \nu_{Q}(\theta)$ as a function of $\theta$. Whereas the zero passage of $\Delta \nu_{Q}(\theta)$ occurs at $\theta=54.7^{\circ}$ for the scenario of a pure angular-dependent $\nu_{Q}^{(L)}$, it is expected at $\theta=64.0(1)^{\circ}$ for the scenario of the large CEFG.

Therefore we measured the angular dependence of the subresonance frequencies in $90^{\circ}$ geometry. Several spectra are shown in Fig. 2. The resonance centers $\nu_{1}$ and $\nu_{2}$ vs $B_{\text {ext }}$ are shown in Fig. 3. All data of the $0^{\circ}$ - and $90^{\circ}$-geometry measurement together were analyzed with a least-squares fit, in which the isotropic and anisotropic parts of the magnetic and the electric hyperfine interaction were free parameters, as well as the demagnetization field and the exact angle $\alpha$ between $c$ and $B_{\text {ext }}$. For each value of $B_{\text {ext }}$ the angle $\theta$ was determined via Eq. (5). Figure 4 shows $\Delta \nu_{Q}$ as function of $\theta$. It is evident that the theoretical curves as obtained from the least-squares fit (full lines in Figs. 3 and 4) describe the experimental data perfectly. The zero passage of $\Delta \nu_{Q}$ occurs at $64.4(1.2)^{\circ}$. The least-squares fit result for the anisotropy constant of the LEFG is $a_{2}^{(L)}=-0.02(7)$. Thus the scenario of an angular-dependent $\nu_{Q}^{(L)}$ can definitely be ruled out, and it

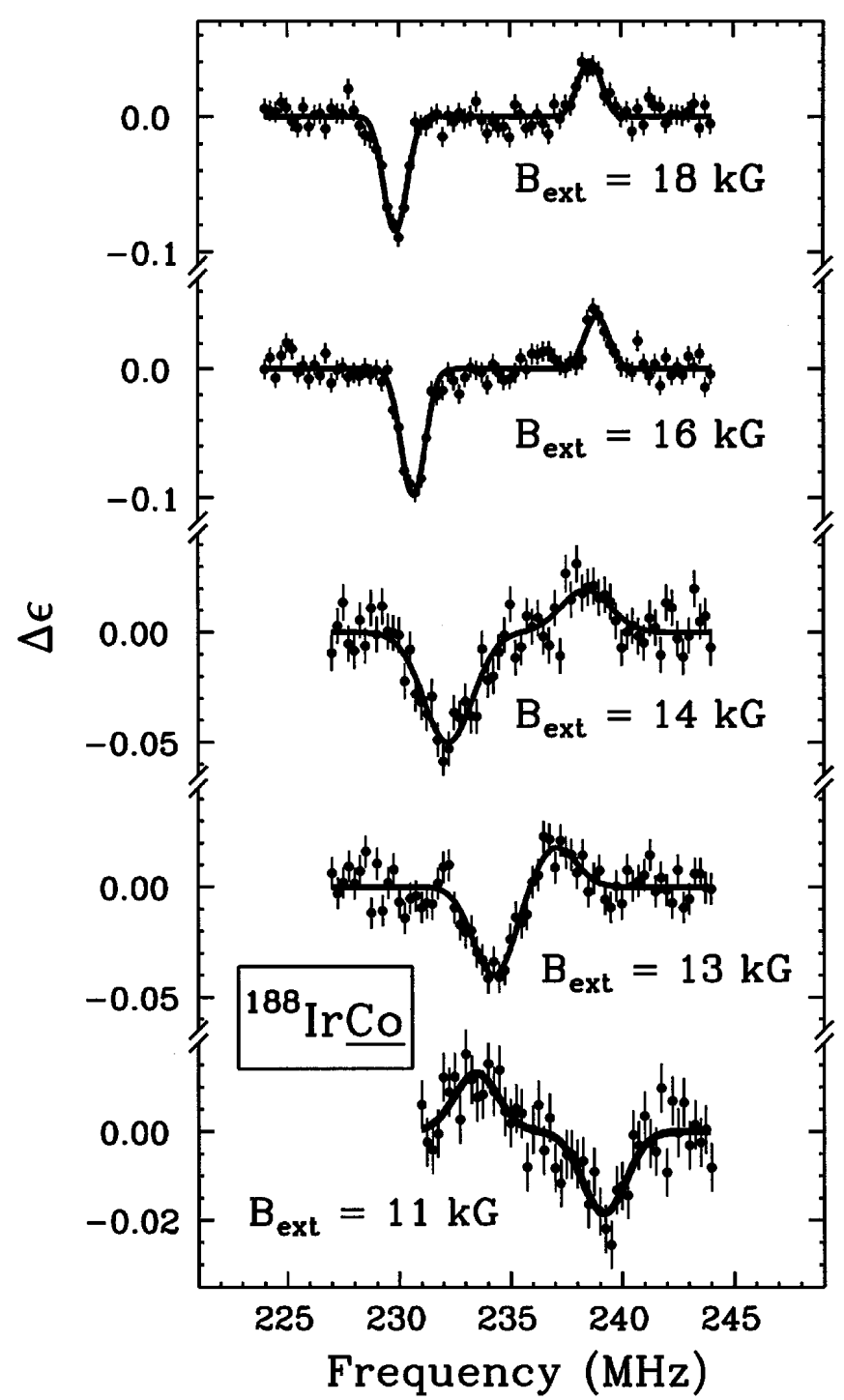

FIG. 2. NMR-ON spectra of ${ }^{188} \mathrm{IrCo} o^{(\mathrm{hcp})}$ measured in $90^{\circ}$ geometry for different values of $B_{\text {ext }}$. It is obvious that the effective quadrupole interaction changes sign between $B_{\text {ext }}=$ 13 and $11 \mathrm{kG}$.

is proven that the strong deviation of $\Delta \nu_{Q}^{\|} / \Delta \nu_{Q}^{\perp}$ from -2 must be due to the existence of a large CEFG.

Taking $Q\left({ }^{188} \mathrm{Ir}\right)=+0.484(6) \mathrm{b}[16]$ and $a_{2}^{(L)}=0$, the final values for the EFG's of Ir in hcp-Co are

$$
\begin{gathered}
e q^{(L)}+e q^{(c, \text { anis })}=-1.790(23) \times 10^{17} \mathrm{~V} / \mathrm{cm}^{2}, \\
e q^{(c, \text { iso })}=-0.378(7) \times 10^{17} \mathrm{~V} / \mathrm{cm}^{2} .
\end{gathered}
$$

The anisotropic part of the CEFG - if existent - cannot be separated experimentally from the LEFG. The result for the isotropic part is, however, unique. In comparison to all known CEFG's of $3 d, 4 d$, and $5 d$ elements in $\mathrm{Fe}, \mathrm{Ni}$, fcc-Co, and hcp-Co the CEFG of $\operatorname{IrCo}{ }^{(\mathrm{hcp})}$ is exceptionally large.

Summarizing, we have investigated the quadrupole interaction of $5 d$ elements in hcp-Co to answer the 


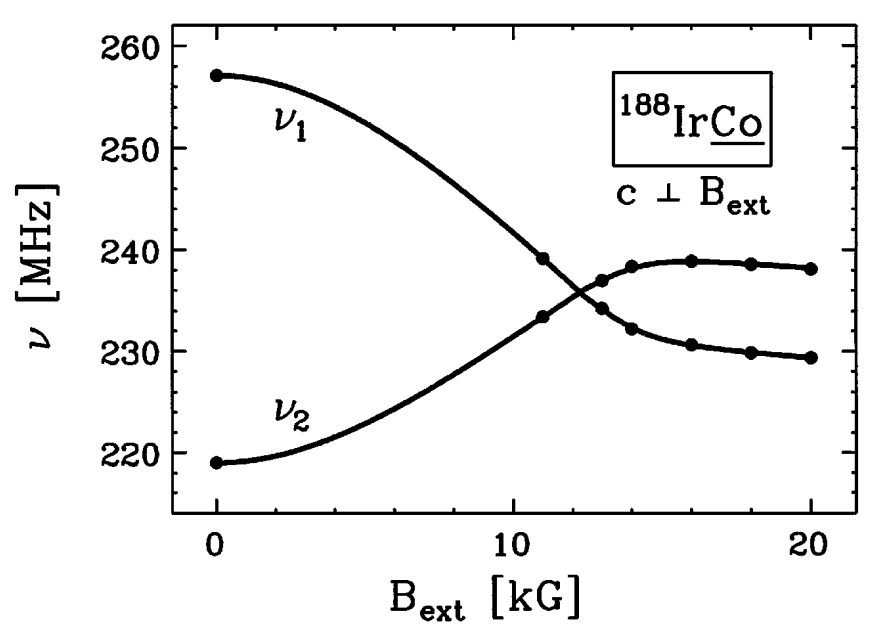

FIG. 3. Dependence on the external magnetic field of the subresonances $\nu_{1}$ and $\nu_{2}$ in $90^{\circ}$ geometry. The solid lines are the result of a least-squares fit. The resonance crossing $\left(\Delta \nu_{Q}=0\right)$ occurs at $B_{\text {ext }}=12.2 \mathrm{kG}$.

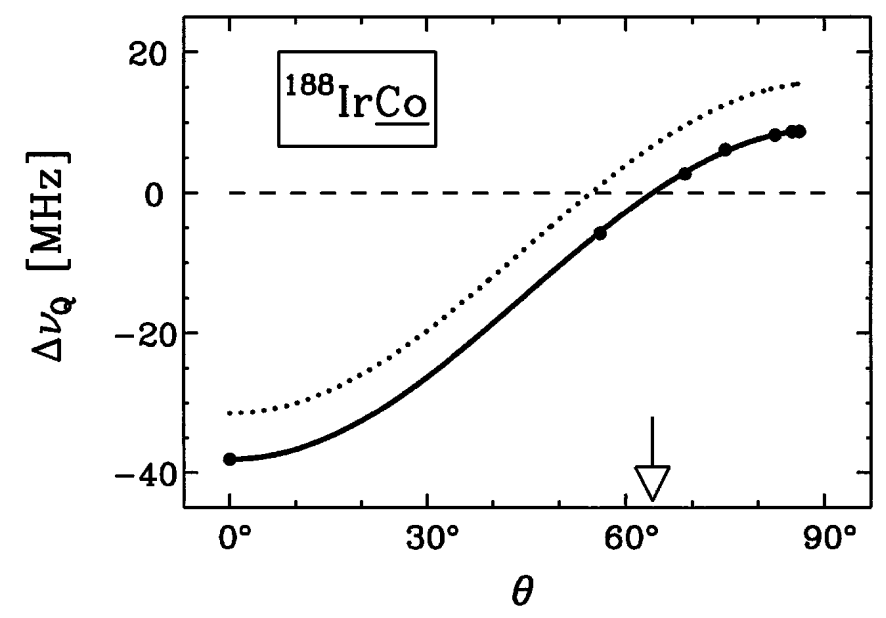

FIG. 4. Dependence of the effective quadrupole splitting on the angle $\theta$ between the electronic magnetization $\vec{M}$ and the single-crystal $c$ axis. The zero passage occurs at $64.4(1.2)^{\circ}$ which proves the existence of a collinear EFG of Ir in hcpCo. The dotted curve represents the lattice contribution to the effective quadrupole splitting (zero passage at $54.7^{\circ}$ ). question whether a collinear EFG acts, similar to those known for the $5 d$ elements in $\mathrm{Fe}, \mathrm{Ni}$, and fcc-Co. The situation for hcp-Co was found to be completely different: Whereas the CEFG of $\mathrm{Pt}, \mathrm{Au}$, and $\mathrm{Hg}$ is very smallthe experimental results are consistent with zero-a very large CEFG was found for Ir in hcp-Co. These findings should stimulate theoretical work on this subject.

We appreciate very much the effort which was put by the ISOLDE and Orsay group into the development of the liquid $\mathrm{Pb}$ target. We also wish to thank Dr. J. Völkl, W. Clauß, G. Neff, H. Schneider, M. Stanger, and H. Utz of the Kristall-Labor for the preparation of the Co single crystal, and E. Smolic for experimental help. This work has been funded by the Deutsche Forschungsgemeinschaft (DFG) under Contract No. Ha 1282/3-3.

[1] M. Aiga and J. Itoh, J. Phys. Soc. Jpn. 31, 1844 (1971).

[2] R. L. Mössbauer, M. Lengsfeld, W.v. Lieres, W. Potzel, P. Teschner, F. E. Wagner, and G. Kaindl, Z. Naturforsch 26A, 343 (1971).

[3] D. Salomon and D. A. Shirley, Phys. Rev. B 9, 29 (1974).

[4] M. Aiga and J. Itoh, J. Phys. Soc. Jpn. 37, 967 (1974).

[5] P. C. Riedi and E. Hagn, Phys. Rev. B 30, 5680 (1984).

[6] G. Seewald et al. (to be published).

[7] M. Kawakami, H. Enokiya, and T. Okamoto, J. Phys. F 15, 1613 (1985).

[8] C. Demangeat, J. Phys. F 4, L64 (1974); 5, 169 (1975).

[9] G. A. Gehring and H.C. W. L. Williams, J. Phys. F 4, 291 (1974).

[10] G. Seewald, E. Hagn, E. Zech, D. Forkel-Wirth, A. Burchard, and ISOLDE Collaboration, Phys. Rev. Lett. 78, 1795 (1997).

[11] H. Enokiya, J. Phys. Soc. Jpn. 42, 796 (1977).

[12] D. Fekete, H. Boasson, A. Grayevski, V. Zevin, and N. Kaplan, Phys. Rev. B 17, 347 (1978).

[13] R. Schmid, B. Hinfurtner, E. Hagn, E. Zech, and M. Deicher, Phys. Lett. A 174, 155 (1993).

[14] E. Matthias and R. J. Holliday, Phys. Rev. Lett. 17, 897 (1966).

[15] K. S. Krane, in Low-Temperature Nuclear Orientation, edited by N.J. Stone and H. Postma (North-Holland, Amsterdam, 1986), p. 31.

[16] G. Seewald, E. Hagn, B. Hinfurtner, E. Zech, D. ForkelWirth, R. Eder, and ISOLDE Collaboration, Phys. Rev. Lett. 77, 5016 (1996). 